\title{
Blow-up and local weak solution for a modified two-component Camassa-Holm equations
}

\author{
Lixin $\operatorname{Tian}^{*}$ and Minyi Zhu
}

* Correspondence: tianlx@ujs.edu. $\mathrm{cn}$

Nonlinear Scientific Research Center, Faculty of Science, Jiangsu University, Zhenjiang, Jiangsu 212013, People's Republic of China

\section{Abstract}

In this article, we establish some blow-up results for a modified two-component Camassa-Holm system in Sobolev spaces. We also obtain the existence of the weak solutions of this system in $H^{s} \times H^{s-1}, s>5 / 2$.

Keywords: the modified two-component Camassa-Holm equations, blow up, weak solutions.

\section{Introduction}

The well-known two-component Camassa-Holm equations [1]

$$
\left\{\begin{array}{l}
m_{t}+2 u_{x} m+u m_{x}+\sigma \rho \rho_{x}=0 \\
\rho_{t}+(u \rho)_{x}=0
\end{array}\right.
$$

where $m=u-u_{x x}$ and $\sigma= \pm 1$. Constantin and Ivanov [2] derived this system in the context of shallow water theory. $u$ can be interpreted as the horizontal fluid velocity and $\rho$ is related to the water elevation in the first approximation $[2,3]$. They showed that while small initial data develop into global solutions, for some initial data wave breaking occurs. They also discussed the solitary wave solutions. In Vlasov plasma models, system (1.1) describes the closure of the kinetic moments of the single-particle probability distribution for geodesic motion on the simplectomorphisms. While in the large-deformation diffeomorphic approach to image matching, system (1.1) is summoned in a type of matching procedure called metamorphosis (see [4] and the references therein). This system appeared originally in [5]. Based on the deformation of biHamiltonian structure of the hydrodynamic type, Chen et al. [6] obtained system (1.1) when $\sigma=-1$. They show that it has the peakon and multilink solitons, and is integrable in the sense that it has Lax-pair. The mathematical properties of system (1.1) have been studied further in many articles, see, e.g., [7-15]. In [4], Holm and Ivanov generalized the Lax-pair formulation of system (1.1) to produce an integrable multi-component family, $\mathrm{CH}(n, k)$, of equations with $n$ components and $1 \leq|k| \leq n$ velocities. They determined their Lie-Poisson Hamiltonian structures and gave numerical examples of their soliton solution behavior. Recently, a new global existence result and several new blow-up results of strong solutions for the Cauchy problem of Equation (1.1) with $\sigma=$ 1 were obtained in [8]. Gui and Liu [14] established the local well posedness for the two-component Camassa-Holm system in a range of the Besov spaces. Chen and Liu

(c) 2012 Tian and Zhu; licensee Springer. This is an Open Access article distributed under the terms of the Creative Commons Attribution License (http://creativecommons.org/licenses/by/2.0), which permits unrestricted use, distribution, and reproduction in any medium, provided the original work is properly cited. 
[16] discussed the wave-breaking phenomenon of a generalized two-component Camassa-Holm system, and determined the exact blow-up rate of such solutions. The existence and uniqueness of global weak solutions to Equation (1.1) have also been discussed by Guan and Yin [17].

In this article, we consider a two-component generalization of Equation (1.1), that is

$$
\left\{\begin{array}{l}
u_{t}-u_{x x t}+3 u^{m} u_{x}-2 u_{x} u_{x x}-u u_{x x x}+\rho \rho_{x}=0 \\
\rho_{t}+(u \rho)_{x}=0
\end{array}\right.
$$

with initial data

$$
\left\{\begin{array}{c}
u(0, x)=u_{0}(x) \in H^{s}, \quad s \geq 1, \quad x \in R, \\
\rho(0, x)=\rho_{0}(x) \in H^{s-1}, \quad s-1 \geq 1, \quad x \in R,
\end{array}\right.
$$

where $m \geq 1$. It can be reduced to (1.1) as $m=1$.

The purpose of this article is to study the well posedness, local weak solution, and blow-up for Cauchy problem (1.2) and (1.3). System (1.2) also conserves conservation laws. Our starting point is to obtain the local well posedness by using Kato's theory, Next, we derive some blow-up results of the solutions by the following transport equation,

$$
\left\{\begin{array}{lr}
q_{t}=u(t, q), & t \in[0, T), \\
q(0, x)=x, & x \in R
\end{array}\right.
$$

which is a crucial ingredient to obtain the blow-up phenomenon. Last, by using the conserves from laws and the contraction mapping theorem, we obtain the existence of weak solutions of Cauchy problem (1.2) and (1.3). These methods are similar to that was used in [18]. However, because of the asymmetry and the high strength of the nonlinearity of Equation (1.3), it is more difficult to estimate the norm of $u, \rho, u_{x} \rho_{x}$ in Sobolev space. In addition, also we get Equation (5.10) which is different with that in [18]. As for the blow-up phenomenon, we get some new results of (1.2) and (1.3).

Guan and Yin $[17,19]$ got the global weak solutions for two-component CamassaHolm shallow water system; they first obtained approximate solutions for the system, then they prove the compactness of these solutions, and at last they got the global weak solutions. Using the same way, Liu and Yin [20] also got global weak solutions for a periodic two-component $\mu$-Hunter-Saxton system. However, in this article, we add high-order perturbation terms in this system, and by using the conserves laws and the contraction mapping theorem, we obtain the existence of weak solutions.

The remainder of this article is organized as follows. Section 2 is the preliminary. In Section 3, the local well posedness for strong solution of Cauchy problem (1.2) and (1.3) is established by Kato's theory. In Section 4, by transport equation, some blow-up results of the solutions of Cauchy problem (1.2) and (1.3) are obtained. The proof of existence of local weak solution is carried out in Section 5 .

\section{Blow-up}

Lemma 2.1: Given $z_{0}=\left(\begin{array}{c}u_{0} \\ \rho_{0}\end{array}\right) \in H^{s} \times H^{s-1}, s>5 / 2$, then there exists a maximal $T=T\left(\left\|z_{0}\right\|_{H^{s} \times H^{s-1}}\right)>0$, and a unique solution $z=\left(\begin{array}{c}u \\ \rho\end{array}\right)$ to Cauchy problem (1.2) and 
(1.3) such that

$$
z\left(\cdot, z_{0}\right) \in C\left([0, T) ; H^{s} \times H^{s-1}\right) \cap C^{1}\left([0, T) ; H^{s-1} \times H^{s-2}\right) .
$$

Moreover, the solution depends continuously on the initial data, i.e., the mapping

$$
z_{0} \rightarrow z\left(\cdot, z_{0}\right): H^{s} \times H^{s-1} \rightarrow C\left([0, T) ; H^{s} \times H^{s-1}\right) \cap C^{1}\left([0, T) ; H^{s-1} \times H^{s-2}\right)
$$

is continuous.

The proof is similar with Theorem 4.1 in [21].

Let $\bar{\rho}=\rho-1$, then (1.2) is equivalent to

$$
\left\{\begin{array}{l}
u_{t}-u_{t x x}+3 u^{m} u_{x}=2 u_{x} u_{x x}+u u_{x x x}-\bar{\rho} \bar{\rho}_{x}-\bar{\rho}_{x} \\
\bar{\rho}_{t}+u \bar{\rho}_{x}=-u_{x} \bar{\rho}_{x}-u
\end{array}\right.
$$

Consider the following initial value problem,

$$
\left\{\begin{array}{lr}
q_{t}=u(t, q), & t \in[0, T) \\
q(0, x)=x, & x \in R
\end{array}\right.
$$

where $u$ is the first component of the solution $z$ to Equation (1.2).

To prove the blow-up result, we need the following lemma.

Lemma 2.2: Let $z_{\alpha} H^{s} \times H^{s-1},(s>5 / 2)$, and let $T>0$ be the maximal existence time of the corresponding solution $z$ to Equation (2.1), then we have

$$
(\bar{\rho}(t, q(t, x))+1) q_{x}(t, x)=\left(\bar{\rho}_{0}(x)+1\right), \forall(t, x) \in[0, T) \times R .
$$

Proof. Differentiating the left-hand side of Equation (2.3) with respect $t$. It follows from (2.1) and (2.2), that

$$
\begin{aligned}
& \frac{d}{d t}(\bar{\rho}(t, q(t, x))+1) q_{x}(t, x) \\
& =\left(\bar{\rho}_{t}(t, q(t, x))+\bar{\rho}_{x}(t, q(t, x)) q_{t}(t, x)\right) q_{x}(t, x)+(\bar{\rho}(t, q(t, x))+1) q_{x t}(t, x) \\
& =\left(\bar{\rho}_{t}(t, q(t, x))+\bar{\rho}_{x}(t, q(t, x)) u(t, q)+\bar{\rho}(t, q) u_{x}(t, q)+u_{x}(t, q)\right) q_{x}(t, x) \\
& =0
\end{aligned}
$$

This completes the proof of this lemma.

Theorem 2.1: Let $z_{0}=\left(\begin{array}{c}u_{0} \\ \bar{\rho}_{0}\end{array}\right) \in H^{s} \times H^{s-1},(s>5 / 2)$, and $T$ be the maximal time of the solution $z$ to Equation (1.2) with the initial data $z_{0}$. Assume that there exists $x_{0} \in$ $R$ such that $\bar{\rho}_{0}\left(x_{0}\right)=-1$ and

$$
u_{0}^{\prime}\left(x_{0}\right)<-\sqrt{2}\left[\left(\left\|u_{0}\right\|_{H^{1}}^{2}+\left\|\rho_{0}\right\|_{L^{2}}^{2}\right)+\frac{3}{m+1}\left(\left\|u_{0}\right\|_{H^{1}}^{2}+\left\|\rho_{0}\right\|_{L^{2}}^{2}\right)^{m+1 / 2}\right]^{1 / 2} .
$$

Then, $T$ is finite and the slope of $u$ tends to negative infinity as $t$ goes to $T$ while $u$ is uniformly bounded on $[0, T)$.

Proof. Let $z=\left(\begin{array}{c}u \\ \bar{\rho}\end{array}\right)$ be the solution of Equation (2.1) with the initial data $z_{0}$, and $T$ be the maximal time of $z$, and let 


$$
n(t)=u_{x}\left(t, q\left(t, x_{0}\right)\right), \gamma(t)=\bar{\rho}\left(t, q\left(t, x_{0}\right)\right)+1
$$

From (2.1) and (2.2), we have

$$
\frac{d n}{d t}=\left(u_{t x}+u u_{x x}\right)\left(t, q\left(t, x_{0}\right)\right), \frac{d \gamma}{d t}=-\gamma n \text {. }
$$

Differentiating the first equation in (2.1) with respect $x$, we have

$$
u_{t x}+u u_{x x}=\frac{3}{m+1} u^{m+1}-\frac{1}{2} u^{2}-\frac{1}{2} u_{x}^{2}+\frac{1}{2} \bar{\rho}^{2}+\bar{\rho}-\Lambda^{-2}\left(\frac{3}{m+1} u^{m+1}-\frac{1}{2} u^{2}+\frac{1}{2} u_{x}^{2}+\frac{1}{2} \bar{\rho}^{2}+\bar{\rho}\right) .
$$

Note that $\gamma(0)=\bar{\rho}_{0}\left(x_{0}\right)+1=0$, Then, by Lemma4.1, we have $\gamma(t)=0, \forall t \in[0, T)$.

Thus

$$
n_{t}=-\frac{1}{2} n^{2}+\frac{1}{2} \gamma^{2}+\frac{3}{m+1} u^{m+1}-\frac{1}{2} u^{2}-\Lambda^{-2}\left(\frac{3}{m+1} u^{m+1}-\frac{1}{2} u^{2}+\frac{1}{2} u_{x}^{2}+\frac{1}{2} \gamma^{2}\right)(t, q) .
$$

Since

$$
\Lambda^{-2}\left(\frac{1}{2} u_{x}^{2}+\frac{1}{2} \gamma^{2}\right) \geq 0
$$

and

$n^{\prime}(t) \leq-\frac{1}{2} n^{2}+u^{2}+\frac{3}{m+1}|u|^{m+1} ., n^{\prime}(t) \leq-\frac{1}{2} n^{2}+u^{2}+\frac{3}{m+1}|u|^{m+1}$.

Note that $\quad\|u\|_{L^{\infty}} \leq\left(\|u\|_{H^{1}}^{2}+\|\rho\|_{L^{2}}^{2}\right)^{1 / 2}=\left(\left\|u_{0}\right\|_{H^{1}}^{2}+\left\|\rho_{0}\right\|_{L^{2}}^{2}\right)^{1 / 2}$. If $\quad$ let $K=\left[\left(\left\|u_{0}\right\|_{H^{1}}^{2}+\left\|\rho_{0}\right\|_{L^{2}}^{2}\right)+\frac{3}{m+1}\left(\left\|u_{0}\right\|_{H^{1}}^{2}+\left\|\rho_{0}\right\|_{L^{2}}^{2}\right)^{m+1 / 2}\right]^{1 / 2}, \quad$ then we have $n^{\prime}(t) \leq-\frac{1}{2} n^{2}(t)+K^{2}$

Since $n(0)<-\sqrt{2} K$, we obtain that $n(t)<-\sqrt{2} t, \forall t \in[0, T)$.

With the inequality above, we get

$$
\frac{n(0)+\sqrt{2} K}{n(0)-\sqrt{2} K} \exp (\sqrt{2} K t)-1 \leq \frac{2 \sqrt{2} K}{n(t)-\sqrt{2} K} \leq 0 .
$$

Since $0<\frac{n(0)+\sqrt{2} K}{n(0)-\sqrt{2} K}<1$, there exists $0<T<\frac{1}{\sqrt{2} K} \ln \left(\frac{n(0)+\sqrt{2} K}{n(0)-\sqrt{2} K}\right)$, such that $\lim _{t \uparrow T} n(t)=-\infty$, i.e., $\lim _{t \uparrow T} u_{x}(t)=-\infty$.

This completes the proof of the theorem.

\section{Local weak solution}

Definition 3.1: $([22])$ Let $\left(u_{0}, \rho_{0}\right) \in H^{1}(R) \times H^{1}(R)$. If $(u, \rho)$ belongs to $L_{\text {loc }}^{\infty}\left([0, T) ; H^{1}(R)\right) \times L_{l o c}^{\infty}\left([0, T) ; H^{1}(R)\right)$ and satisfies the identity

$$
\int_{0}^{T} \int_{R}\left(z \psi_{t}+F(u) \psi_{x}\right) d x d t+\int_{R} z_{0}(x) \psi(0, x) d x=0
$$

for $\quad$ all $\quad \psi \in C_{c}^{\infty}([0, T) \times R) \times C_{c}^{\infty}([0, T) \times R), \quad$ where $\psi \in C_{c}^{\infty}([0, T) \times R) \times C_{c}^{\infty}([0, T) \times R)$, the set of all the restrictions to $([0, T) \times R) \times$ $([0, T) \times R)$ of smooth functions on $R^{2} \times R^{2}$ with compact support contained in $((-T, T)$ $\times R) \times((-T, T) \times R)$. Then, $z$ is called a weak solution to Equation (1.6). If $z$ is a weak 
solution on $[0, T) \times[0, T)$ for every $T>0$, then it is called global weak solution to Equation (1.6).

In this section, we discuss the existence of weak solution of Cauchy problem (1.2) and (1.3). To this purpose, we consider the following Cauchy problem:

$$
\begin{aligned}
& \left\{\begin{array}{l}
u_{t}-u_{x x t}+\varepsilon u_{x x x x}+3 u^{m} u_{x}-2 u_{x} u_{x x}-u u_{x x x}+\rho \rho_{x}=0, \\
\rho_{t}+\varepsilon \rho_{x x t}+(u \rho)_{x}=0
\end{array}\right. \\
& \begin{cases}u(0, x)=u_{0}(x) \in H^{s}, & s \geq 1, \quad x \in R . \\
\rho(0, x)=\rho_{0}(x) \in H^{s-1}, & s-1 \geq 1, \quad x \in R .\end{cases}
\end{aligned}
$$

where $\varepsilon$ is a constant satisfying $0<\varepsilon<1 / 4$. Note that when $\varepsilon=0$, system (3.1) and (3.2) is just the system (1.2) and (1.3).

For any $0<\varepsilon<1 / 4$ and $s \geq 1$, the integral operators

$$
D_{1}=\left(1-\partial_{x}^{2}+\varepsilon \partial_{x}^{4}\right)^{-1}: H^{s} \rightarrow H^{s+4}
$$

and

$$
D_{2}=\left(1+\varepsilon \partial_{x}^{2}\right)^{-1}: H^{s} \rightarrow H^{s+2}
$$

define two bounded linear operator in the indicated Sobolev spaces.

To prove the existence of solutions to the problem (3.1) and (3.2), we apply the two operators above to both sides of (3.1) and then integrate the resulting equations with regard to $t$. This leads to the following equations.

$$
\left\{\begin{array}{l}
u(x, t)=u_{0}(x)+\int_{0}^{t} D_{1}\left[\left(-\frac{3}{m+1} u^{m+1}\right)_{x}+\left(u_{x}^{2}\right)_{x}+\left(u u_{x x}-\frac{1}{2} u_{x}^{2}\right)_{x}+\frac{1}{2}\left(\rho^{2}\right)_{x}\right] d \tau . \\
\rho(x, t)=\rho_{0}(x)+\int_{0}^{t} D_{2}\left[-(u \rho)_{x}\right] d \tau
\end{array}\right.
$$

A standard application of the contraction mapping theorem leads to the following existence result.

Theorem 3.1: For each initial data $u_{0} \in H^{s}(s \geq 1), \rho_{0} \in H^{s-1}(s \geq 2)$, there exists a $T$ $>0$ depending only on the norm of $\left\|u_{0}\right\|_{H^{s}},\left\|\rho_{0}\right\|_{H^{s-1}}$, and $m$ such that there exists a unique solution $(u, \rho) \in C\left([0, T] ; H^{s}\right) \times C\left([0, T] ; H^{s-1}\right)$ of system (3.1) and (3.2) in the sense of distribution. If $u_{0} \in H^{s}(s \geq 2), \rho_{0} \in H^{s-1}(s \geq 3)$, the solution $(u, \rho) \in C([0, \infty]$; $\left.H^{s}\right) \times C\left([0, \infty] ; H^{s-1}\right)$ exists for all time, in particular, when $u_{0} \in H^{s}(s \geq 4), \rho_{0} \in H^{s-1}(s$ $\geq 5$ ), the corresponding solution is a classical globally defined solution of (3.1) and (3.2).

The global existence result follows from the conservation law

$$
\int_{R}\left(u^{2}+\rho^{2}+u_{x}^{2}+\varepsilon \rho_{x}^{2}+\varepsilon u_{x x}^{2}\right) d x=\int_{R}\left(u_{0}^{2}+\rho_{0}^{2}+u_{0 x}^{2}+\varepsilon \rho_{0 x}^{2}+\varepsilon u_{0 x x}^{2}\right) d x
$$

admitted by (3.1) in its integral form.

Theorem 3.2: Suppose that for some $s \geq 4$, the function pair $u(x, t)$ and $\rho(x, t)$ in the solution of Equation (3.1) corresponding to the initial data $u_{0} \in H^{s}(s \geq 4) ; \rho_{0} \in H^{s-1}(s$ $\geq 5$ ), then the following inequalities hold: 


$$
\|u\|_{H^{1}}^{2},\|\rho\|_{L^{2}}^{2} \leq \int_{R}\left(u_{0}^{2}+\rho_{0}^{2}+u_{0 x}^{2}+\varepsilon \rho_{0 x}^{2}+\varepsilon u_{0 x x}^{2}\right) d x .
$$

For any real number $q \in(1, s](s \geq 5)$, there exists a constant $c$ depending only on $q$, $m$, such that

$$
\begin{aligned}
& \int_{R}\left[(1-\varepsilon)\left(\Lambda^{q} u\right)^{2}+\left(\Lambda^{q-1} \rho\right)^{2}+(1+\varepsilon)\left(\Lambda^{q-1} \rho_{x}\right)^{2}+\varepsilon\left(\Lambda^{q} u_{x}\right)^{2}+\varepsilon\left(\Lambda^{q-1} u\right)^{2}\right] d x \leq \\
& \int_{R}\left[(1-\varepsilon)\left(\Lambda^{q} u_{0}\right)^{2}+\left(\Lambda^{q-1} \rho_{0}\right)^{2}+(1+\varepsilon)\left(\Lambda^{q-1} \rho_{0 x}\right)^{2}+\varepsilon\left(\Lambda^{q} u_{0 x}\right)^{2}+\varepsilon\left(\Lambda^{q-1} u_{0}\right)^{2}\right] d x \\
& +c \int_{0}^{t}\left(\|u\|_{L^{\infty}}^{m}+\left\|\rho_{x}\right\|_{L^{\infty}}+\left\|u_{x}\right\|_{L^{\infty}}+\|\rho\|_{L^{2}}\right)\left(\|u\|_{H^{q}}^{2}+\|\rho\|_{H^{q-1}}^{2}\right) d \tau .
\end{aligned}
$$

For any $q \in(1, s-1](s \geq 4)$, there exists a constant $c$ such that

$$
(1-\varepsilon)\left\|u_{t}\right\|_{H^{q}} \leq c\left(1+\left\|u_{x}\right\|_{L^{\infty}}+\left\|\rho_{x}\right\|_{L^{\infty}}\right)\left(\|u\|_{H^{q}}+\|u\|_{H^{q}}^{m}+\|\rho\|_{H^{q-1}}\right) .
$$

And for any $q \in(1, s-2](s \geq 5)$, there exists a constant $c$ such that

$$
\left\|\rho_{t}\right\|_{H^{q-1}} \leq c\left(1+\left\|u_{x}\right\|_{L^{\infty}}+\left\|\rho_{x}\right\|_{L^{\infty}}\right)\left(\|u\|_{H^{q}}+\|u\|_{H^{q}}^{m}+\|\rho\|_{H^{q-1}}\right) .
$$

Proof. It is obvious that (3.3) holds. In order to prove (5.4), let $\Lambda=\left(1-\partial_{x}^{2}\right)^{1 / 2}$. We rewrite Equation (3.1) in the following equivalent form.

$$
\left\{\begin{array}{l}
(1-\varepsilon) u_{t}-\varepsilon u_{x x t}+\varepsilon \Lambda^{-2} u_{t}=-\Lambda^{-2}\left(\rho \rho_{x}+3 u^{m} u_{x}-2 u_{x} u_{x x}-u u_{x x x}\right) \\
\rho_{t}+(1+\varepsilon) \Lambda^{-2} \rho_{x x t}=-\Lambda^{-2}(u \rho)_{x}
\end{array}\right.
$$

For any $q \in(1, s](s \geq 5)$, applying $\left(\Lambda^{q} u\right) \Lambda^{q}$ to the both sides of the first equation of Equation (3.7), respectively, and integrating with regard to $x$, we obtain

$$
\begin{aligned}
& (1-\varepsilon)\left(\Lambda^{q} u, \Lambda^{q} u_{t}\right)-\varepsilon\left(\Lambda^{q} u, \Lambda^{q} u_{x x t}\right)+\varepsilon\left(\Lambda^{q} u, \Lambda^{q-2} u_{t}\right) \\
& =\frac{1}{2} \frac{d}{d t} \int_{R}\left[(1-\varepsilon)\left(\Lambda^{q} u\right)^{2}+\varepsilon\left(\Lambda^{q} u_{x}\right)^{2}+\varepsilon\left(\Lambda^{q-1} u\right)^{2}\right] d x
\end{aligned}
$$

By using Sobolev embedding theorems, we have

$$
\begin{aligned}
& \left|\left(\Lambda^{q} u, \Lambda^{q-2} \rho \rho_{x}\right)_{0}\right| \leq\left|\left(\left[\Lambda^{q-2}, \rho\right] \rho_{x}, \Lambda^{q} u\right)_{0}\right|+\left|\left(\rho \Lambda^{q-2} \rho_{x}, \Lambda^{q} u\right)_{0}\right| \\
& \leq c\|u\|_{H^{q}}\left(\left\|\rho_{x}\right\|_{L^{\infty}}\left\|\Lambda^{q-3} \rho_{x}\right\|_{L^{2}}+\left\|\Lambda^{q-2} \rho\right\|_{L^{2}}\left\|\rho_{x}\right\|_{L^{\infty}}\right)+\|u\|_{H^{q}}\|\rho\|_{L^{2}}\left\|\Lambda^{q-2} \rho_{x}\right\|_{L^{2}} \\
& \leq c\|u\|_{H^{q}}\left(\left\|\rho_{x}\right\|_{L^{\infty}}\|\rho\|_{H^{q-2}}+\|\rho\|_{H^{q-2}}\left\|\rho_{x}\right\|_{L^{\infty}}+\|\rho\|_{L^{2}}\|\rho\|_{H^{q-1}}\right) \\
& \leq c\left(\left\|\rho_{x}\right\|_{L^{\infty}}+\|\rho\|_{L^{2}}\right)\left(\|u\|_{H^{q}}^{2}+\|\rho\|_{H^{q-1}}^{2}\right),
\end{aligned}
$$

where we have used lemma in [23] with $r=q-2>0$. Also

$$
\begin{aligned}
& \left|\left(\Lambda^{q} u, \Lambda^{q-2}\left(3 u^{m} u_{x}-2 u_{x} u_{x x}-u u_{x x x}\right)\right)_{0}\right|=\left|\left(\partial_{x} \Lambda^{-2}\left(\frac{3}{m+1} u^{m+1}-\frac{1}{2} u_{x}^{2}-u u_{x x}\right), u\right)_{q}\right| \\
& \leq\left\|\frac{3}{m+1} u^{m+1}-\frac{1}{2} u_{x}^{2}-u u_{x x}\right\|_{H^{q-1}}\|u\|_{H^{q}} \leq c\left(\left\|u^{m}\right\|_{L^{\infty}}+\left\|u_{x}\right\|_{L^{\infty}}\right)\left(\|u\|_{H^{q}}^{2}+\|\rho\|_{H^{q-1}}^{2}\right),
\end{aligned}
$$

where we have used Lemma in [24] with $r=q-1>0$. 
Then, we get

$$
\begin{aligned}
& \frac{1}{2} \frac{d}{d t} \int_{R}\left[(1-\varepsilon)\left(\Lambda^{q} u\right)^{2}+\varepsilon\left(\Lambda^{q} u_{x}\right)^{2}+\varepsilon\left(\Lambda^{q-1} u\right)^{2}\right] d x \\
& \leq c\left(\|u\|_{L^{\infty}}^{m}+\left\|u_{x}\right\|_{L^{\infty}}+\left\|\rho_{x}\right\|_{L^{\infty}}+\|\rho\|_{L^{2}}\right)\left(\|u\|_{H^{q}}^{2}+\|\rho\|_{H^{q-1}}^{2}\right) .
\end{aligned}
$$

For any $q \in(1, s-1](s \geq 5)$, applying $\left(\Lambda^{q-1} \rho\right) \Lambda^{q-1}$ to the both sides of the second equation of Equation (3.7), respectively, then we obtain

$$
\frac{1}{2} \frac{d}{d t} \int_{R}\left[\left(\Lambda^{q-1} \rho\right)^{2}+(1+\varepsilon)\left(\Lambda^{q-2} \rho_{x}\right)^{2}\right] d x \leq c\left(\|u\|_{L^{\infty}}+\|\rho\|_{L^{\infty}}\right)\left(\|u\|_{H^{q}}^{2}+\|\rho\|_{H^{q-1}}^{2}\right) .
$$

Summing up (3.8) and (3.9), we get

$$
\begin{aligned}
& \int_{R}\left[(1-\varepsilon)\left(\Lambda^{q} u\right)^{2}+\left(\Lambda^{q-1} \rho\right)^{2}+(1+\varepsilon)\left(\Lambda^{q-2} \rho_{x}\right)^{2}+\varepsilon\left(\Lambda^{q} u_{x}\right)^{2}+\varepsilon\left(\Lambda^{q-1} u\right)^{2}\right] d x \\
& \leq \int_{R}\left[(1-\varepsilon)\left(\Lambda^{q} u_{0}\right)^{2}+\left(\Lambda^{q-1} \rho_{0}\right)^{2}+(1+\varepsilon)\left(\Lambda^{q-2} \rho_{0 x}\right)^{2}+\varepsilon\left(\Lambda^{q} u_{0 x}\right)^{2}+\varepsilon\left(\Lambda^{q-1} u_{0}\right)^{2}\right] d x \\
& +c \int_{0}^{t}\left(\|u\|_{L^{\infty}}^{m}+\left\|\rho_{x}\right\|_{L^{\infty}}+\left\|u_{x}\right\|_{L^{\infty}}+\|\rho\|_{L^{2}}\right)\left(\|u\|_{H^{q}}^{2}+\|\rho\|_{H^{q-1}}^{2}\right) d \tau .
\end{aligned}
$$

For any $q \in(1, s-1](s \geq 4)$, applying $\left(\Lambda^{q} u_{t}\right) \Lambda^{q}$ to the both sides of the first equation of Equation (3.7), respectively, and integrating with regard to $x$, we obtain that

$$
(1-\varepsilon)\left(\Lambda^{q} u_{t}, \Lambda^{q} u_{t}\right)-\varepsilon\left(\Lambda^{q} u_{t}, \Lambda^{q} u_{x x t}\right)+\varepsilon\left(\Lambda^{q} u_{t}, \Lambda^{q-2} u_{t}\right)=(1-\varepsilon)\left\|u_{t}\right\|_{H^{q}}^{2}+\varepsilon\left\|u_{x t}\right\|_{H^{q}}^{2}+\varepsilon\left\|u_{t}\right\|_{H^{q-1}}^{2},
$$

and

$$
\begin{aligned}
& \left|\left(\partial_{x} \Lambda^{-2} \rho^{2}, u_{t}\right)_{q}\right|=\left|\left(u_{t}, \Lambda^{-2} \rho \rho_{x}\right)_{q}\right| \leq\left|\left(\left[\Lambda^{q-2}, \rho\right] \rho_{x}, \Lambda^{q} u_{t}\right)_{0}\right|+\left|\left(\rho \Lambda^{q-2} \rho_{x}, \Lambda^{q} u_{t}\right)_{0}\right| \\
& \leq c\left\|u_{t}\right\|_{H^{q}}\left(\left\|\rho_{x}\right\|_{L^{\infty}}\left\|\Lambda^{q-3} \rho_{x}\right\|_{L^{2}}+\left\|\Lambda^{q-2} \rho\right\|_{L^{2}}\left\|\rho_{x}\right\|_{L^{\infty}}+\|\rho\|_{L^{2}}\left\|\Lambda^{q-2} \rho_{x}\right\|_{L^{2}}\right) \\
& \leq c\left\|u_{t}\right\|_{H^{q}} \cdot\|\rho\|_{H^{q-2}}\left(\left\|\rho_{x}\right\|_{L^{\infty}}+\|\rho\|_{L^{2}}\right), \\
& \left|\left(u_{t}, \Lambda^{-2}\left(3 u^{m} u_{x}-2 u_{x} u_{x x}-u u_{x x x}\right)\right)_{q}\right|=\left|\left(u_{t}, \partial_{x} \Lambda^{-2}\left(\frac{3}{m+1} u^{m+1}-\frac{1}{2} u_{x}^{2}-u u_{x x}\right)\right)_{q}\right| \\
& \leq\left\|u_{t}\right\|_{H^{q}} \cdot\left\|\frac{3}{m+1} u^{m+1}-\frac{1}{2} u_{x}^{2}-u u_{x x}\right\|_{H^{q-1}} \\
& \leq c\left\|u_{t}\right\|_{H^{q}}\left(\|u\|_{L^{\infty}}^{m}+\|u\|_{L^{\infty}}+\left\|u_{x}\right\|_{L^{\infty}}\right)\left(\|u\|_{H^{q}}+\|u\|_{H^{q}}^{m}\right),
\end{aligned}
$$

where we have used lemma in [24] with $r=q-1>0$. Then, we get

$$
(1-\varepsilon)\left\|u_{t}\right\|_{H^{q}} \leq c\left(1+\left\|u_{x}\right\|_{L^{\infty}}+\left\|\rho_{x}\right\|_{L^{\infty}}\right)\left(\|u\|_{H^{q}}+\|u\|_{H^{q}}^{m}+\|\rho\|_{H^{q-1}}\right) .
$$

For any $q \in(1, s-2](s \geq 5)$, applying $\left(\Lambda^{q-1} \rho_{t}\right) \Lambda^{q-1}$ to the both sides of the second equation of Equation (5.7), respectively, then we obtain

$$
\begin{aligned}
& \left\|\rho_{t}\right\|_{H^{q-1}} \leq c\left(1+\left\|\rho_{x}\right\|_{L^{\infty}}+\left\|u_{x}\right\|_{L^{\infty}}\right)\left(\|u\|_{H^{q}}+\|\rho\|_{H^{q-1}}\right) \\
& \leq c\left(1+\left\|\rho_{x}\right\|_{L^{\infty}}+\left\|u_{x}\right\|_{L^{\infty}}\right)\left(\|u\|_{H^{q}}+\|u\|_{H^{q}}^{m}+\|\rho\|_{H^{q-1}}\right) .
\end{aligned}
$$

This complete the proof of the theorem.

Suppose $u_{0} \in H^{s}(s \geq 1), \rho_{0} \in H^{s-1}(s \geq 2)$, and let $u_{\varepsilon 0}, \rho_{\varepsilon 0}$ be the convolution $u_{\varepsilon 0}=$ $\phi_{\varepsilon}^{*} u_{0}, \rho_{\varepsilon 0}=\phi_{\varepsilon}^{*} \rho_{0}$, where $\varphi_{\varepsilon}(x)=\varepsilon^{-1 / 4} \cdot \hat{\varphi}\left(\varepsilon^{-1 / 4 x}\right)$ such that the Fourier transform $\hat{\varphi}$ of $\phi$ satisfies $\hat{\varphi} \in C_{0}^{\infty}, \hat{\varphi}(\xi) \geq 0$, and $\hat{\varphi}(\xi)=1$ for any $\xi \in(-1,1)$. Then, it follows 
from Theorem 3.1 that for each $\varepsilon$ with $0<\varepsilon<1 / 4$, the Cauchy problem

$$
\begin{aligned}
& \left\{\begin{array}{r}
u_{t}-u_{x x t}+\varepsilon u_{x x x x t}+3 u^{m} u_{x}-2 u_{x} u_{x x}-u u_{x x x}+\rho \rho_{x}=0 \\
\rho_{t}+\varepsilon \rho_{x x t}+(u p)_{x}=0
\end{array}\right. \\
& \left\{\begin{array}{l}
u(0, x)=u_{\varepsilon 0}(x), \quad t \geq 0, \quad x \in R \\
\rho(0, x)=\rho_{\varepsilon 0}(x), \quad t \geq 0, \quad x \in R
\end{array}\right.
\end{aligned}
$$

has a unique solution $u_{\varepsilon}(t, x) \in C^{\infty}\left([0, \infty) ; H^{\infty}\right.$ and $\rho_{\varepsilon}(t, x) \in C^{\infty}\left([0, \infty) ; H^{\infty}\right.$. We first demonstrate the properties of the initial data $u_{\varepsilon 0}, \rho_{\varepsilon 0}$ in the following lemma. The proof is similar to Lemma 5 in [25].

Lemma 3.1: Under the above assumptions, there hold

$$
\begin{aligned}
& \left\|u_{\varepsilon 0}\right\|_{H^{q}} \leq c \text {, if } q \leq s ;\left\|\rho_{\varepsilon 0}\right\|_{H^{q-1}} \leq c \text {, if } q-1 \leq s .
\end{aligned}
$$

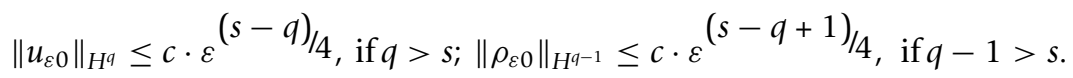

for any $\varepsilon$ with $0<\varepsilon<1 / 4$, where $c$ is a constant independent of $\varepsilon$. The proof is similar to Lemma 5 in [25].

Theorem 3.3: Suppose that $u_{0}(x) \in H^{s}(R), s \in[1,3 / 2] ; \rho_{0}(x) \in H^{s-1}(R)$,

$s-1 \in[1,3 / 2]$ such that $\left\|u_{0 x}\right\|_{L^{\infty}}<\infty,\left\|\rho_{0 x}\right\|_{L^{\infty}}<\infty$. Let $u_{\varepsilon 0}=\phi_{\varepsilon}{ }^{*} u_{0}, \rho_{\varepsilon 0}=\phi_{\varepsilon}{ }^{*} \rho_{0}$, be defined the same as above. Then, there exist constants $T>0$ and $c>0$ independent of $\varepsilon$ such that the corresponding solution $u_{\varepsilon}, \rho_{\varepsilon}$ of (3.10) satisfy the inequalities $\left\|\rho_{\varepsilon x}\right\|_{L^{\infty}} \leq c,\left\|\rho_{\varepsilon x}\right\|_{L^{\infty}} \leq c$ for any $t \in[0, T)$.

Proof. Use Equation (3.7) with $u=u_{\varepsilon}, \rho=\rho_{\varepsilon}$. Differentiating with respect to $x$ on both sides of the first equation in Equation (3.7). Note that $\partial_{x}^{2} \Lambda^{-2}=\Lambda^{-2}-I$, we obtain

$$
\begin{aligned}
& (1-\varepsilon) u_{x t}-\varepsilon u_{x x x t}=-\frac{1}{2} \Lambda^{-2} \rho^{2}+\frac{1}{2} \rho^{2}-\partial_{x}^{2} \Lambda^{-2}\left(\frac{3}{m+1} u^{m+1}-\frac{1}{2} u_{x}^{2}-u u_{x x}\right)-\varepsilon \Lambda^{-2} u_{x t} \\
& =\frac{1}{2} \rho^{2}+\frac{3}{m+1} u^{m+1}-\frac{1}{2} u_{x}^{2}-u u_{x x}-\Lambda^{-2}\left(\frac{1}{2} \rho^{2}+\frac{3}{m+1} u^{m+1}-\frac{1}{2} u_{x}^{2}-u u_{x x}\right)-\varepsilon \Lambda^{-2} u_{x t}
\end{aligned}
$$

Let $n>0$ be an integer. Then, multiplying the above equation by $\left(u_{x}\right)^{2 \mathrm{n}+1}$ to integrate with respect to $x$, we get

$$
\begin{aligned}
& \int_{R}\left[(1-\varepsilon) u_{x t}\left(u_{x}\right)^{2 n+1}-\varepsilon u_{x x x t}\left(u_{x}\right)^{2 n+1}\right] d x \\
& =\int_{R} \frac{1}{2} \rho^{2}\left(u_{x}\right)^{2 n+1} d x+\int_{R} \frac{3}{m+1} u^{m+1}\left(u_{x}\right)^{2 n+1} d x-\int_{R} \frac{1}{2}\left(u_{x}\right)^{2 n+3} d x+\frac{1}{2 n+2} \int_{R}\left(u_{x}\right)^{2 n+3} d x-\int_{R} g\left(u_{x}\right)^{2 n+1} d x,
\end{aligned}
$$

where $g=\Lambda^{-2}\left(\frac{1}{2} \rho^{2}+\frac{3}{m+1} u^{m+1}-\frac{1}{2} u_{x}^{2}-u u_{x x}\right)+\varepsilon \partial_{x} \Lambda^{-2} u_{t}$.

It follows from Hölder inequality that

$$
\begin{aligned}
& (1-\varepsilon) \frac{d}{d t}\left(\int_{R}\left(u_{x}\right)^{2 n+2} d x\right)^{\frac{1}{2 n+2}} \leq \varepsilon\left(\int_{R}\left|u_{x x x t}\right|^{2 n+2} d x\right)^{\frac{1}{2 n+2}}+\frac{1}{2}\left(\int_{R} \rho^{4 n+4} d x\right)^{\frac{1}{2 n+2}} \\
& +\frac{3}{m+1}\left(\int_{R}\left(u^{m+1}\right)^{2 n+2} d x\right)^{\frac{1}{2 n+2}}+\frac{1}{2}\left(\int_{R}\left|u_{x}\right|^{4 n+4} d x\right)^{\frac{1}{2 n+2}}+\frac{1}{2 n+2}\left(\int_{R} u_{x}^{4 n+4} d x\right)^{\frac{1}{2 n+2}}+\left(\int_{R}|g|^{2 n+2} d x\right)^{\frac{1}{2 n+2}} .
\end{aligned}
$$


Note that $\|f\|_{L^{p}} \rightarrow\|f\|_{L^{\infty}}$ as $p \rightarrow \infty$ for any $f \in L^{\infty} \cap L^{2}$. Integrating the above inequality over $R$ with respect to $t$, and taking the limitation as $n \rightarrow \infty$, we have

$$
(1-\varepsilon)\left\|u_{x}\right\|_{L^{\infty}} \leq(1-\varepsilon)\left\|u_{0 x}\right\|_{L^{\infty}}+\int_{0}^{t}\left[\varepsilon\left\|u_{x x x t}\right\|_{L^{\infty}}+c\left(\left\|\rho^{2}\right\|_{L^{\infty}}+\left\|u^{m+1}\right\|_{L^{\infty}}+\left\|u_{x}^{2}\right\|_{L^{\infty}}+\|g\|_{L^{\infty}}\right)\right] d \tau .
$$

It follows from (3.3) that

$$
\|g\|_{L^{\infty}} \leq \tilde{c}\left(\left\|u_{t}\right\|_{L^{2}}+\|\rho\|_{L^{2}}^{2}+\|u\|_{L^{2}}^{m+1}+\left\|u_{x}\right\|_{L^{2}}^{2}+\left\|u u_{x x}\right\|_{L^{2}}\right) \leq \tilde{c}_{1}\left(1+\left\|u_{t}\right\|_{L^{2}}+\left\|u u_{x x}\right\|_{L^{2}}\right) .
$$

For any given $r \in(1 / 2,1)$, we have

$$
\left\|u_{x x x t}\right\|_{L^{\infty}} \leq c_{r}\left\|u_{x x x t}\right\|_{H^{r}} \leq c_{r}\left\|u_{t}\right\|_{H^{r+3}} \leq c_{r}\left(1+\left\|u_{x}\right\|_{L^{\infty}}+\left\|\rho_{x}\right\|_{L^{\infty}}\right)\left(\|u\|_{H^{r+4}}+\|u\|_{H^{r+4}}^{m}+\|\rho\|_{H^{r+3}}\right) .
$$

Then from (3.4), we have

$$
\begin{aligned}
& \|u\|_{H^{r+4}}+\|\rho\|_{H^{+3}} \leq \sqrt{2}\left(\|u\|_{H^{r+4}}^{2}+\|\rho\|_{H^{r+3}}^{2}\right)^{2} \leq \sqrt{\frac{2}{1-\varepsilon}}\left\{(1-\varepsilon)\left\|u_{0}\right\|_{H^{r+4}}^{2}+\varepsilon\left\|u_{0 x}\right\|_{H^{r^{r+4}}}^{2}+\varepsilon\left\|u_{0}\right\|_{H^{+3}}^{2}\right. \\
& \left.+\left\|\rho_{0}\right\|_{H^{+3}}^{2}+(1+\varepsilon)\left\|\rho_{0 x}\right\|_{H^{+3}}^{2}\right\}^{1 / 2} \cdot \exp \left[c \int\left(\|u\|_{L^{\infty}}^{m}+\|\rho\|_{L^{2}}+\left\|u_{x}\right\|_{L^{\infty}}+\left\|\rho_{x}\right\|_{L^{\infty}}\right) d \tau\right] \\
& \leq c \varepsilon^{(s-r-4) / 4} \cdot \exp \left[c \int_{0}^{t}\left(1+\left\|u_{x}\right\|_{L^{\infty}}+\left\|\rho_{x}\right\|_{L^{\infty}}\right) d \tau\right]
\end{aligned}
$$

and

$$
\|u\|_{H^{r+4}}+\|u\|_{H^{r+4}}^{m}+\|\rho\|_{H^{r+3}} \leq\left(\|u\|_{H^{r+4}}+\|\rho\|_{H^{r+3}}\right)+\left(\|u\|_{H^{r+4}}+\|\rho\|_{H^{r+3}}\right)^{m}
$$

Thus,

$$
\begin{aligned}
& \left\|u_{x x x t}\right\|_{L^{\infty}} \leq c_{r} \varepsilon^{(s-r-4) / 4}\left(1+\left\|u_{x}\right\|_{L^{\infty}}+\left\|\rho_{x}\right\|_{L^{\infty}}\right) \cdot \exp \left[c \int_{0}^{t}\left(1+\left\|u_{x}\right\|_{L^{\infty}}+\left\|\rho_{x}\right\|_{L^{\infty}}\right) d \tau\right] \\
& +c_{m} \varepsilon^{(s-r-4) m / 4}\left(1+\left\|u_{x}\right\|_{L^{\infty}}+\left\|\rho_{x}\right\|_{L^{\infty}}\right)^{m} \cdot \exp \left[c m \int_{0}^{t}\left(1+\left\|u_{x}\right\|_{L^{\infty}}+\left\|\rho_{x}\right\|_{L^{\infty}}\right) d \tau\right]
\end{aligned}
$$

and

$$
\begin{aligned}
& \left\|\rho^{2}\right\|_{L^{\infty}} \leq\|\rho\|_{L^{\infty}}^{2} \leq\|\rho\|_{H^{1}}^{2}=\int_{R}|\rho|^{2} d x+\int_{R}\left|\rho_{x}\right|^{2} d x=\|\rho\|_{L^{2}}^{2}+\left\|\rho_{x}\right\|_{L^{2}}^{2} \leq c\left(1+\left\|\rho_{x}\right\|_{L^{2}}^{2}\right) \leq c\left(1+\left\|\rho_{x}\right\|_{L^{\infty}}^{2}\right) \\
& \left\|u^{m+1}\right\|_{L^{\infty}} \leq\|u\|_{L^{\infty}}^{m+1} \leq\|u\|_{H^{1}}^{m+1} \leq c, \\
& \left\|u_{t}\right\|_{L^{2}} \leq\left\|u_{t}\right\|_{H^{1}} \leq\left\|u_{t}\right\|_{H^{r+3}} \leq\left(1+\left\|u_{x}\right\|_{L^{\infty}}+\left\|\rho_{x}\right\|_{L^{\infty}}\right) \cdot\left(\|u\|_{H^{r+4}}+\|u\|_{H^{r+4}}^{m}+\|\rho\|_{H^{r+4}}\right) \\
& \left\|u u_{x x}\right\|_{L^{2}} \leq\left\|u u_{x x}\right\|_{H^{1}} \leq c\left(\|u\|_{L^{\infty}}\left\|u_{x x}\right\|_{H^{1}}+\|u\|_{H^{1}}\left\|u_{x x}\right\|_{L^{\infty}}\right) \leq c\|u\|_{H^{1}}\|u\|_{H^{r+4}} \leq c\|u\|_{H^{r+4}} .
\end{aligned}
$$

Then, we get

$$
\|g\|_{L^{\infty}} \tilde{\leq} \tilde{c}\left[\left(1+\left(1+\left\|u_{x}\right\|_{L^{\infty}}+\left\|\rho_{x}\right\|_{L^{\infty}}\right)\right) \cdot\left(\|u\|_{H^{r+4}}+\|u\|_{H^{r+4}}^{m}+\|\rho\|_{H^{r+3}}\right)\right] .
$$

It follows that

$$
\begin{aligned}
& \left\|u_{x}\right\|_{L^{\infty}} \leq\left\|u_{0_{x}}\right\|_{L^{\infty}}+\frac{1}{1-\varepsilon} \int_{0}^{t}\left\{c \left[1+\left(\left\|u_{x}\right\|_{L^{\infty}}+\left\|\rho_{x}\right\|_{L^{\infty}}\right)+\left(\left\|u_{x}\right\|_{L^{\infty}}+\left\|\rho_{x}\right\|_{L^{\infty}}\right)^{2}+\left(1+\left\|u_{x}\right\|_{L^{\infty}}+\left\|\rho_{x}\right\|_{L^{\infty}}\right)\right.\right. \\
& \left.\left.\cdot\left(\|u\|_{H^{r+4}}+\|u\|_{H^{r+4}}^{m}+\|\rho\|_{H^{+3}}\right)\right]+\varepsilon c_{r}\left(1+\left\|u_{x}\right\|_{L^{\infty}}+\left\|\rho_{x}\right\|_{L^{\infty}}\right) \cdot\left(\|u\|_{H^{r^{+4}}}+\|u\|_{H^{r+4}}^{m}+\|\rho\|_{H^{r^{*}}}\right)\right\} d \tau \\
& \leq\left\|u_{0_{x}}\right\|_{L^{\infty}}+\frac{4 c}{3} \int_{0}^{t}\left\{1+\left(\left\|u_{x}\right\|_{L^{\infty}}+\left\|\rho_{x}\right\|_{L^{\infty}}\right)+\left(\left\|u_{x}\right\|_{L^{\infty}}+\left\|\rho_{x}\right\|_{L^{\infty}}\right)^{2}+\left(1+\left\|u_{x}\right\|_{L^{\infty}}+\left\|\rho_{x}\right\|_{L^{\infty}}\right)\right.
\end{aligned}
$$


$\left.\exp \left[c \int_{0}^{\tau}\left(1+\left\|u_{x}\right\|_{L^{\infty}}+\left\|\rho_{x}\right\|_{L^{\infty}}\right) d \eta\right]+\left(1+\left\|u_{x}\right\|_{L^{\infty}}+\left\|\rho_{x}\right\|_{L^{\infty}}\right)^{m} \cdot \exp \left[c m \int_{0}^{\tau}\left(1+\left\|u_{x}\right\|_{L^{\infty}}+\left\|\rho_{x}\right\|_{L^{\infty}}\right) d \eta\right]\right\} d \tau$, where $c$ is a constant depends on $\Lambda^{-2}$ and $m$.

Also, we can obtain

$$
\begin{aligned}
& \left\|\rho_{x}\right\|_{L^{\infty}} \leq\left\|\rho_{0 x}\right\|_{L^{\infty}}+c \int_{0}^{t}\left\{1+\left(\left\|u_{x}\right\|_{L^{\infty}}+\left\|\rho_{x}\right\|_{L^{\infty}}\right)+(1+\varepsilon)\left(1+\left\|u_{x}\right\|_{L^{\infty}}+\left\|\rho_{x}\right\|_{L^{\infty}}\right)\right. \\
& \left.\left(\|u\|_{H^{r+4}}+\|u\|_{H^{r+4}}^{m}+\|\rho\|_{H^{r+3}}\right)\right\} d \tau,
\end{aligned}
$$

where $r \in\left(\frac{1}{2}, 1\right)$. From (3.9), we derive

$$
\begin{aligned}
& \left\|u_{x}\right\|_{L^{\infty}}+\left\|\rho_{x}\right\|_{L^{\infty}} \leq\left\|u_{0 x}\right\|_{L^{\infty}}+\left\|\rho_{0 x}\right\|_{L^{\infty}}+\frac{4 c}{3} \int_{0}^{t}\left\{1+\left(\left\|u_{x}\right\|_{L^{\infty}}+\left\|\rho_{x}\right\|_{L^{\infty}}\right)\right. \\
& +\left(\left\|u_{x}\right\|_{L^{\infty}}+\left\|\rho_{x}\right\|_{L^{\infty}}\right)^{2}+\left(1+\left\|u_{x}\right\|_{L^{\infty}}+\left\|\rho_{x}\right\|_{L^{\infty}}\right) \cdot \exp \left[c \int_{0}^{\tau}\left(1+\left\|u_{x}\right\|_{L^{\infty}}+\left\|\rho_{x}\right\|_{L^{\infty}}\right) d \eta\right] \\
& \left.+\left(1+\left\|u_{x}\right\|_{L^{\infty}}+\left\|\rho_{x}\right\|_{L^{\infty}}\right)^{m} \cdot \exp \left[c m \int_{0}^{\tau}\left(1+\left\|u_{x}\right\|_{L^{\infty}}+\left\|\rho_{x}\right\|_{L^{\infty}}\right) d \eta\right]\right\} d \tau .
\end{aligned}
$$

It follows from the contraction mapping theorem that there exists a constant $T>0$ such that the equation

$$
\begin{aligned}
& f(t)=\left\|u_{0 x}\right\|_{L^{\infty}}+\left\|\rho_{0 x}\right\|_{L^{\infty}}+\frac{4 c_{m}}{3} \int_{0}^{t}\left\{\left[1+f(\tau)+f^{2}(\tau)+(1+f(\tau))\right.\right. \\
& \left.\exp \left[c \int_{0}^{\tau}(1+f(\eta)) d \eta\right]+(1+f(\tau))^{m} \cdot \exp \left[c m \int_{0}^{\tau}(1+f(\eta)) d \eta\right]\right\} d \tau,
\end{aligned}
$$

has a unique solution $f(t) \in C[0, T]$. Theorem II in Section I.1 in [26] shows that $\left\|u_{x}\right\|_{L^{\infty}}+\left\|\rho_{x}\right\|_{L^{\infty}} \leq f(t)$ for any $t \in[0, T]$ which leads to the conclusion of this theorem.

Let $u=u_{\varepsilon} \rho=\rho_{\varepsilon}$, with (3.4) used

$$
\left\|u_{\varepsilon}\right\|_{H^{q}}+\left\|\rho_{\varepsilon}\right\|_{H^{q-1}} \leq c \varepsilon^{(s-r-4) / 4} \cdot \exp \left[c \int_{0}^{t}\left(1+\left\|u_{x}\right\|_{L^{\infty}}+\left\|\rho_{x}\right\|_{L^{\infty}}\right) d \tau\right] \leq c \cdot \exp \left[c \int_{0}^{t}(1+f(\tau)) d \tau\right]
$$

where $s \in\left[1, \frac{3}{2}\right], r \in\left(\frac{1}{2}, 1\right)$.

$$
\begin{aligned}
& \left\|u_{\varepsilon t}\right\|_{H^{r}}+\left\|\rho_{\varepsilon t}\right\|_{H^{r-1}}=\left\|u_{t}\right\|_{H^{r}}+\left\|\rho_{t}\right\|_{H^{r-1}} \leq c(1+f(t)) \cdot \exp \left[c \int_{0}^{t}(1+f(\tau)) d \tau\right] \\
& +c(1+f(t))^{m} \cdot \exp \left[c m \int_{0}^{t}(1+f(\tau)) d \tau\right],
\end{aligned}
$$

where $q \in(0, \mathrm{~s}], r \in(0, \mathrm{~s}-1], t \in[0, T]$.

Then, it follows from Aubin's compactness theorem [27] that there exist subsequences of $\left\{u_{\varepsilon}\right\},\left\{\rho_{\varepsilon}\right\}$ denoted by $\left\{u_{\varepsilon_{n}}\right\},\left\{\rho_{\varepsilon_{n}}\right\}$ such that $\left\{u_{\varepsilon_{n}}\right\},\left\{\rho_{\varepsilon_{n}}\right\}$ are weakly convergent to $u(t, x) \in L^{2}\left([0, T] ; H^{s}\right), \rho(t, x) \in L^{2}\left([0, T] ; H^{s-1}\right)$, respectively, and $\left\{u_{\varepsilon_{n}} t\right\},\left\{\rho_{\varepsilon_{n}}\right\}$ are weakly convergent to $u_{t}(t, x) \in L^{2}\left([0, T] ; H^{s-1}\right), \rho_{t}(t, x) \in L^{2}\left([0, T] ; H^{s-2}\right)$, respectively. Because $\left\{u_{\varepsilon_{n}}\right\}$ are weakly convergent to $u(t, x) \in L^{2}\left([0, T] ; H^{s}\right), f\left(u_{\varepsilon_{n}}-u\right) \rightarrow 0$ for any $f \in\left(L^{2}\left([0, T] ; H^{s}\right)\right)^{*}=L^{2}\left([0, T] ; H^{s}\right)$ when $n \rightarrow \infty$. Applying Riesz lemma, we conclude that there exists $f_{u_{\varepsilon n}-u}$ such that 


$$
f_{u_{\varepsilon_{n}}-u}\left(u_{\varepsilon_{n}}-u\right)=\left(u_{\varepsilon_{n}}-u, u_{\varepsilon_{n}}-u\right)=\left\|u_{\varepsilon_{n}}-u\right\|^{2} .
$$

Since $f_{u_{\varepsilon_{n}}-u}\left(u_{\varepsilon_{n}}-u\right) \rightarrow 0$ as $n \rightarrow \infty$, we have $\left\|u_{\varepsilon_{n}}-u\right\|^{2} \rightarrow 0$. Then for any real $R$ $>0,\left\{u_{\varepsilon_{n}}\right\}$ converges strongly to $u \in L^{2}\left([0, T] ; H^{q}(-R, R)\right)$ for any $q \in[0, s-1)$; and $\left\{u_{\varepsilon_{n}}\right\}$ converges to $u_{t}$ strongly in $L^{2}\left([0, T] ; H^{r}(-R, R)\right)$ for any $r \in[0, s-1]$. Similarly, $g\left(\rho_{\varepsilon_{n}}-\rho\right) \rightarrow 0$ for any $g \in\left(L^{2}\left([0, T] ; H^{s}\right)\right)^{*}=L^{2}\left([0, T] ; H^{s}\right)$ as $n \rightarrow \infty$. By Riesz lemma, we conclude that there exists $g_{u_{\varepsilon n}}-u$ such that

$$
g_{u_{\varepsilon_{n}}-u}\left(u_{\varepsilon_{n}}-u\right)=\left(u_{\varepsilon_{n}}-u, u_{\varepsilon_{n}}-u\right)=\left\|u_{\varepsilon_{n}}-u\right\|^{2} .
$$

Since $g_{u_{\varepsilon_{n}}-u}\left(u_{\varepsilon_{n}}-u\right) \rightarrow 0$ as $n \rightarrow \infty$, we have $\left\|u_{\varepsilon_{n}}-u\right\|^{2} \rightarrow 0$. Then for any real $R$ $>0,\left\{u_{\varepsilon_{n}}\right\}$ converges strongly to $\rho \in L^{2}\left([0, T] ; H^{q-1}(-R, R)\right)$ for any $q \in[0, s-1)$, and $\left\{\rho_{\varepsilon_{n}} t\right\}$ converge to $u_{b} \rho_{t}$ strongly in $L^{2}\left([0, T] ; H^{r-1}(-R, R)\right)$ for any $r \in[0, s-1]$. Hence, the existence of a weak solution to the Cauchy problem (1.2) and (1.3) is established.

Theorem 3.4: Let $u_{0}(x) \in H^{s}(R)(s \in[1,3 / 2])$ and $\rho_{0}(x) \in H^{s-1}(R)(s-1 \in[1,3 / 2])$, which satisfy $\left\|u_{0 x}\right\|_{L^{\infty}}<\infty,\left\|\rho_{0 x}\right\|_{L^{\infty}}<\infty$. Then there exists a constant $T>0$ such that the Cauchy problem (1.2) and (1.3) with the initial data has a solution

$$
(u(t, x), \rho(t, x)) \in L^{2}\left([0, T] ; H^{s}\right) \times L^{2}\left([0, T] ; H^{s-1}\right),
$$

in the sense of distribution. And $u_{\infty} \rho_{x} \in L^{\infty}([0, T] \times R)$.

Proof. It follows from Theorem 3.3 that $\left\{u_{\varepsilon_{n} x}\right\},\left\{\rho_{\varepsilon_{n} x}\right\}$ are bounded in the space $L^{\infty}$. Hence, the sequences $\left\{u_{\varepsilon_{n}}^{2}\right\},\left\{\rho_{\varepsilon_{n}}^{2}\right\},\left\{u_{\varepsilon_{n} x}^{2}\right\},\left\{\rho_{\varepsilon_{n} x}^{2}\right\},\left\{u_{\varepsilon_{n}} \rho_{\varepsilon_{n}}\right\},\left\{u_{\varepsilon_{n} x} \rho_{\varepsilon_{n} x}\right\},\left\{u_{\varepsilon_{n} x} \rho_{\varepsilon_{n}}\right\}$, $\left\{u_{\varepsilon_{n}} \rho_{\varepsilon_{n} x}\right\}$ are also weakly convergent to $u^{2}, \rho^{2}, u_{x}^{2}, \rho_{x}^{2}, u \rho, u_{x} \rho_{x}, u_{x} \rho, u \rho_{x} \in L^{2}\left([0, T] ; H^{r}\right.$ $(-R, R))$ for any $r \in[0, s-1]$ and $R>0$, respectively. Therefore, $u, \rho$ satisfy

$$
\int_{0}^{T} \int_{R} u\left(f_{t}-f_{x x t}\right) d x d t=-\int_{0}^{T} \int_{R}\left[\left(\frac{3}{m+1} u^{m+1}+\frac{1}{2} u_{x}^{2}+\frac{1}{2} \rho^{2}\right) f_{x}+u u_{x} f_{x x}\right] d x d t,
$$

and

$$
\int_{0}^{T} \int_{R} \rho f_{t} d x d t=-\int_{0}^{T} \int_{R}(u \rho) f d x d t
$$

with $u(0, x)=u_{0}(x), \rho(0, x)=\rho_{0}(x)$, and any $f \in C_{0}^{\infty}$. Moreover, since $X=L^{1}([0, T] \times$ $R)$ is a separable Banach space and $\left\{u_{\varepsilon_{n} x}\right\},\left\{\rho_{\varepsilon_{n}} x\right\}$ are bounded sequences in the dual space $X^{*}=L^{\infty}([0, T] \times R)$ of $X$, there are two subsequences of $\left\{u_{\varepsilon_{n} x}\right\},\left\{\rho_{\varepsilon_{n} x}\right\}$ (still denoted by $\left.\left\{u_{\varepsilon_{n} x}\right\},\left\{\rho_{\varepsilon_{n} x}\right\}\right)$ weak star convergent to two functions $U, P \in L^{\infty}([0, T] \times$ $R)$, respectively. Because $\left\{u_{\varepsilon_{n} x}\right\},\left\{\rho_{\varepsilon_{n} x}\right\}$ are also weakly convergent to $u_{\infty} \rho_{x} \in L^{\infty}([0, T]$ $\times R$ ), respectively. It follows that $u_{x}=U, \rho_{x}=P$ hold almost everywhere. Hence, $u_{x}, \rho_{x}$ $\in L^{\infty}([0, T] \times R)$.

\section{Acknowledgements}

The study was supported by the National Nature Science Foundation of China (No. 11171135, 71073072), the Nature Science Foundation of Jiangsu (No. BK 2010329), the Project of Excellent Discipline Construction of Jiangsu Province of China, and the Natural Science Foundation of the Jiangsu Higher Education Institutions of China (No. 09KJB110003). 


\section{Authors' contributions}

LT raised the modified two-component Camassa-Holm equations and conceived the local weak solution of the equations. MZ carried out the solution of the weak solution and its blow-up phenomenon. All authors read and approved the final manuscript.

\section{Competing interests}

The authors declare that they have no competing interests.

\section{Received: 14 November 2011 Accepted: 2 May 2012 Published: 2 May 2012}

\section{References}

1. Ivanov, R: Extended Camassa-Holm hierarchy and conserved quantities. Z Naturforsch A. 61, 133-138 (2006)

2. Constantin, A, Ivanov, Rl: On an integrable two-component Camassa-Holm shallow water system. Phys Lett A. 372, 7129-7132 (2008)

3. Ivanov, R: Two-component integrable systems modelling shallow water waves. Oberwolfach Rep. 6, 429-462 (2009)

4. Holm, DD, Ivanov, RI: Multi-component generalizations of the $\mathrm{CH}$ equation: geometrical aspects, peakons and numerical examples. J Phys A 43, 492001 (2010). doi:10.1088/1751-8113/43/49/492001

5. Olver, P, Rosenau, P: Tri-Hamiltonian duality between solitons and solitary-wave solutions having compact support. Phys Rev E. 53, 1900-1906 (1996)

6. Chen, M, Liu, SQ, Zhang, Y: A 2-component generalization of the Camassa-Holm equation and its solutions. Lett Math Phys. 75, 1-15 (2006)

7. Holm, DD, Náraigh, LÓ, Tronci, C: Singular solutions of a modified two-component CH equation. Phys Rev E. 79, 016601 (2009)

8. Liu, SQ, Zhang, Y: Deformations of semisimple bi-hamiltonian structures of hydrodynamic type. J Geom Phys. 54 427-453 (2005)

9. Chen, RM, Liu, Y: Wave breaking and global existence for a generalized two-component Camassa-Holm. Int Math Res Notes. 6, 1381-1416 (2011)

10. Henry, D: Infinite propagation speed for a two component Camassa-Holm equation. Discr Contin Dyn Syst Ser B. 12, 597-606 (2009)

11. Zhang, P, Liu, Y: Stability of solitary waves and wave-breaking phenomena for the two-component CH system. Int Math Res Notes. 11, 1981-2021 (2010)

12. Guan, C, Yin, ZY: Global existence and blow-up phenomena for an integrable two-component Camassa-Holm shallow water system. J Diff Equ. 248, 2003-2014 (2010)

13. Gui, G, Liu, Y: On the global existence and wave-breaking criteria for the two-component Camassa-Holm system. J Funct Anal. 258, 4251-4278 (2010)

14. Gui, G, Liu, Y: On the Cauchy problem for the two-component Camassa-Holm system. Math Z. 268, 45-66 (2010)

15. Escher, J: Well-posedness and blow-up phenomena for the 2-component Camassa-Holm equation. Discr Contin Dyn Syst. 19, 493-513 (2007)

16. Chen, RM, Liu, Y: Wave-breaking and global existence for a generalized two-component Camassa-Holm system. Math Phys Sci Int Math Res Notes. 6, 1381-1416 (2011)

17. Guan, C, Yin, Z: Global weak solutions for a two-component Camassa-Holm shallow water system. J Funct Anal. 260, 1132-1154 (2011)

18. Fu, Y, Qu, C: Well posedness and blow-up solution for a new coupled Camassa-Holm equations with peakons. J Math Phys 50, 012906 (2009). doi:10.1063/1.3064810

19. Guan, C, Yin, Z: Global weak solutions for a modified two-component Camassa-Holm shallow water system. Ann I.H. Poincare-AN (2011). doi:10.1016/j.anihpc.2011.04.003

20. Liu, J, Yin, Z: Global weak solutions for a periodic two-component $\mu$-Hunter-Saxton system. Math AP 3, arxXiv (2011). $1012.5452 v 3$

21. $\mathrm{Fu}, \mathrm{Y}, \mathrm{Liu}, \mathrm{Y}, \mathrm{Qu}, \mathrm{C}$ : Well-posedness and blow-up solution for a modified two-component periodic Camassa-Holm system with peakons. Math Ann. 348, 415-448 (2000)

22. Zhang, S, Yin, Z: Global weak solutions for the Dullin-Gottwald-Holm equation. Nonlinear Anal. 72, 1690-1700 (2010)

23. Kato, T, Ponce, G: Communtator estimates and the Euler and Navier-Stokes equations. Commun Pure Appl Math. 41, 891-907 (1988)

24. Kato, T: On the Korteweg-de Vries equation. Manuscr Math. 28, 89-99 (1979)

25. Constantin, A: Existence of permanent and breaking waves for a shallow water equation. A geometric approach Ann Inst Fourier (Grenoble). 50, 321-362 (2000)

26. Water, W: Differential and Integral Inequalities. pp. 362. Springer-Verlag, New York55, (1970)

27. Lions, JL: Quelques méthodes de résolution des problemes aux limites non linéaires, Gauthier-Villars, Paris. 574 (1969)

doi:10.1186/1687-2770-2012-52

Cite this article as: Tian and Zhu: Blow-up and local weak solution for a modified two-component Camassa-

Holm equations. Boundary Value Problems 2012 2012:52. 